CARDIOVASCULAR MEDICINE

\title{
Spectrum of cardiac abnormalities associated with long QT in stroke survivors
}

\author{
K Y K Wong, S McSwiggan, N S J Kennedy, S Y S Wong, A Gavin, R S MacWalter, \\ A D Struthers
}

See end of article for authors' affiliations

......................

Correspondence to: Dr Kenneth Wong, Cardiothoracic CentreLiverpool NHS Trust, Thomas Drive, Liverpool L14 3PE, UK; kywong@ doctors.org.uk

Accepted 3 November 2004
Objectives: To find out what spectrum of cardiac abnormalities are found in those stroke survivors who can be deemed to be at high cardiac risk by their having long QT.

Methods: 202 patients with good recovery from a cerebrovascular event occurring at least one month previously were recruited into a prospective epidemiological study. These stroke survivors underwent a battery of cardiac investigations including 12 lead ECG, echocardiography, myocardial perfusion scanning, and heart rate variability assessment. The ECGs were digitised by a single observer blinded to the blood pressure and other investigations of the patients. The maximum heart rate corrected QT interval (QTc max) in the 12 lead ECG was derived by Bazett's formula.

Results: Prolonged QTc max significantly correlated with increasing blood pressure, left ventricular mass index, and depressed heart rate variability. As the number of cardiac abnormalities increased, QTc max became more prolonged.

Conclusions: Long QT is significantly associated with left ventricular mass index even after adjustment for both systolic and diastolic blood pressures. Long QT was also associated with the total cardiac disease burden. These two observations may explain why stroke survivors with long QTc max were at greater risk of cardiac death.
A fter surviving a stroke, more people die of cardiac causes than of a recurrent stroke. QT prolongation was recently shown to predict cardiac death in stroke survivors. ${ }^{1}$ Similar findings have been seen in many other populations. This raises the question of why QT prolongation predicts cardiac death. Two possibilities arise although they are not necessarily exclusive of each other. Firstly, QT prolongation may be a marker of treatable, hidden but potentially lethal cardiac abnormalities, such as ischaemia or left ventricular abnormalities. Secondly, QT prolongation may be a marker of arrhythmogenicity, irrespective of ischaemia or left ventricular abnormalities.

The main objective of this prospective study was to test the hypothesis that maximum heart rate corrected QT interval (QTc max) prolongation is a convenient marker of treatable, hidden cardiac abnormalities in stroke survivors. If so, this may contribute strongly to its adverse prognostic significance.

\section{METHODS}

Two hundred and two patients with good recovery after a recently documented stroke or transient ischaemic attack occurring at least one month previously were studied. A random sample of the stroke outpatient clinic was recruited and this comprised $70 \%$ who had been hospitalised within 72 hours after their acute cerebrovascular event plus 30\% who had community strokes. Patients who had atrial fibrillation or flutter, bigeminy, paced rhythm, or bundle branch block on their ECG were excluded from QT interval analysis. (These ECG changes make the QT interval difficult to measure reliably.) Other exclusion criteria were inability to provide consent, for example, because of dementia; age greater than 90 years; and being under nursing home-type care.

The study was approved by the Tayside committee on medical research ethics. All participants gave informed consent to the study. The procedures followed were in accordance with institutional guidelines. The study also had administration of radioactive substances advisory committee approval for myocardial perfusion scanning.

A focused history and examination were undertaken for all the patients, noting in particular any common risk factors of cerebrovascular disease and ischaemic heart disease.

The patients then underwent thorough cardiac investigations including resting ECG, echocardiography, stress myocardial perfusion study, and ambulatory ECG monitoring.

\section{Resting ECG}

Resting supine 12 lead ECGs (Marquette Medical Systems, Milwaukee, Wisconsin, USA) were recorded at $20 \mathrm{~mm} / \mathrm{mV}$ and $25 \mathrm{~mm} / \mathrm{s}$.

ECGs were analysed by one investigator who was blinded to other investigation findings, including patients' blood pressure. QT intervals were analysed by digitising the ECGs. The technique of digitising ECGs we used was first described in 1994. ${ }^{2}$ The QT interval was defined as the time between the onset of the QRS complex and the end of the T wave (when the $\mathrm{T}$ wave returned to the isoelectric line). If the $\mathrm{T}$ wave was followed by a $\mathrm{U}$ wave then the nadir between the $\mathrm{T}$ and the $\mathrm{U}$ wave (that is, the lowest point of the curve) was taken as the point where the T wave ended. QTc was defined as the QT interval divided by the square root of the RR interval (Bazett's formula). The QTc max was defined as the maximum QTC measured from a 12 lead ECG.

Abbreviations: BSA, body surface area; HOPE, heart outcomes prevention evaluation; HRV, heart rate variability; IVSd, interventricular septal thickness in end diastole; LIFE, losartan intervention for end point reduction in hypertension; LVH, left ventricular hypertrophy; LVIDd, end diastolic left ventricular internal dimension; LVMI, left ventricular mass index; PROGRESS, perindopril protection against recurrent stroke study; PWTd, posterior wall thickness in end diastole; QTc max, maximum heart rate corrected QT interval; SDNN, standard deviation of normal to normal RR intervals; UK-HEART, UK-heart failure evaluation and assessment of risk trial 


\section{Echocardiography}

Transthoracic echocardiography was performed with the Hewlett Packard SONOS 2000 phased array imaging system (Andover, Massachusetts, USA).

\section{Left ventricular mass index estimations}

Interventricular septal thickness in end diastole (IVSd), end diastolic left ventricular internal dimension (LVIDd), and left ventricular posterior wall thickness in end diastole (PWTd) were measured from $\mathrm{M}$ mode measurements obtained at the level of the papillary muscles from parasternal views. ${ }^{3}$ Left ventricular mass index (LVMI) was calculated with the American Society of Echocardiography guidelines and measurements were made from leading edge to leading edge, as follows: $\mathrm{LVMI}=\left(0.83 \times\left[(\text { LVIDd+PWTd+IVSd })^{3}-(\text { LVIDd })^{3}\right]\right.$ $-0.6 \mathrm{~g}) / \mathrm{BSA}$, where BSA is body surface area in square metres, calculated as $0.0001 \times 71.84 \times$ weight $(\mathrm{kg})^{0.425} \times$ height $(\mathrm{cm})^{0.725}$.

Left ventricular hypertrophy $(\mathrm{LVH})$ was defined as LVMI $\geqslant 134 \mathrm{~g} / \mathrm{m}^{2}$ in men and $\geqslant 110 \mathrm{~g} / \mathrm{m}^{2}$ in women.

Assessment of LV systolic function

The method of discs volume calculation was based on area traces of the left ventricle from the apical four chamber view.

\section{Stress myocardial perfusion study for myocardial ischaemia}

Myocardial perfusion studies were carried out with dipyridamole. Dipyridamole was slowly infused at $0.56 \mathrm{mg} / \mathrm{kg}$ over about four minutes. This was followed by simple arm exercise for around two minutes. The patient was then injected with $400 \mathrm{MBq}$ of 99m-technetium tetrofosmin (Amersham Health, Little Chalfont, Buckinghamshire, UK). The stress scan was carried out 45 minutes after injection with a dual head Philips Axis gamma camera (Philips Medical Systems) fitted with high resolution collimators. If the stress scan was abnormal, images obtained after an injection at rest (about one week later) were used to differentiate between infarction and ischaemia.

The scans were ranked as normal or abnormal by an experienced observer (NSJK) blinded to the QT data. In addition to the qualitative assessment, the scans were assessed quantitatively. Each scan was scored out of 64 by dividing the heart into 16 regions (four apical, three anterior, three lateral, three inferior, and three septal) and giving each region a score out of 4 , indicating the degree of perfusion. The inducible ischaemia score was defined as the difference between the score obtained from the stress scan and that of the rest scan (that is, rest scan score minus stress scan score). An inducible ischaemia score $\geqslant 3$ was regarded as evidence of significant inducible myocardial ischaemia.

Patients were excluded from this part of the study if they had moderate to severe asthma or severe chronic obstructive pulmonary disease and were taking theophylline medication.

\section{Ambulatory ECG monitoring}

Twenty four hour ambulatory ECGs were recorded (upgraded Reynolds Pathfinder 700 series, Reynolds Medical Limited, Hertford, UK) for assessment of heart rate variability (HRV). Information was collected on the time domain parameters from the whole 24 hour tapes with methods recommended by the European Society of Cardiology and the North American Society of Pacing and Electrophysiology in $1996 .{ }^{4}$ Patients with atrial fibrillation or paroxysmal atrial fibrillation during the 24 hour ECG monitoring were excluded from HRV analysis.

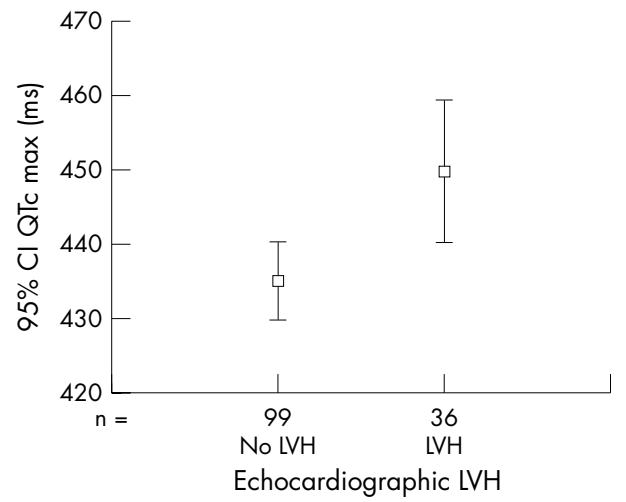

Figure 1 Relation between echocardiographic left ventricular hypertrophy (LVH) and maximum heart rate corrected QT interval (QTc max).

\section{Statistical analysis}

SPSS (SPSS Inc, Chicago, Illinois, USA) was used for statistical analyses. Univariate analysis of variance and Spearman non-parametric tests were used to test the relation between long QT (QTc max) and various cardiac abnormalities.

\section{RESULTS}

\section{Patient characteristics}

Two hundred and two stroke survivors were recruited. The mean (SD) age of the cohort of stroke survivors was 66 (9.4) years (range 30-84 years). Their mean (SD) resting clinic blood pressure was 145/80 (21.2/11.0) $\mathrm{mm} \mathrm{Hg}$ (range 82/48 to $220 / 125 \mathrm{~mm} \mathrm{Hg}$ ). There were 122 male patients $(60.4 \%)$. Mean (SD) weight was 79 (15.8) $\mathrm{kg}$ and $12.2 \%$ had diabetes. Their mean (SD) total cholesterol was $4.92 \mathrm{mmol} / \mathrm{l}$ and mean (SD) high density lipoprotein cholesterol was 1.33 (0.41) $\mathrm{mmol} / \mathrm{l}$. At the time of the study $22.8 \%$ were smokers and $50.5 \%$ were former smokers. Of strokes for which there was computed tomography or magnetic resonance imaging evidence, $82 \%$ were infarcts and $18 \%$ were bleeds. Thirty six per cent of patients had transient ischaemic attacks. Mean (SD) QTc max was 440 (29) ms. The range of QTc max in our cohort was $377 \mathrm{~ms}$ to $530 \mathrm{~ms}$. Ninety two per cent of patients were not taking drugs that would prolong the QT interval. Nine per cent of patients had potassium $<3.5 \mathrm{mmol} / \mathrm{l}$ and $38 \%$ of patients were taking diuretics at the time of the study.

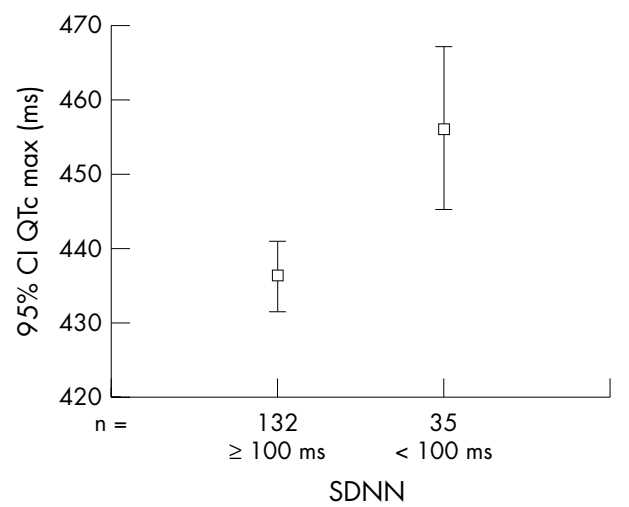

Figure 2 Relation between QTc max and abnormal heart rate variability (as indexed by moderately depressed standard deviation of normal to normal RR intervals (SDNN) $<100 \mathrm{~ms}$ ). 


\section{QTc max and blood pressure}

QTc max was weakly albeit highly significantly correlated with both systolic (Spearman $r=0.3$, two tailed $\mathrm{p}<0.001$ ) and diastolic blood pressures (Spearman $r=0.2$, two tailed $\mathrm{p}=0.007)$.

\section{QTc max and LVH}

Twenty six per cent (37 of 141) of patients had LVH (fig 1). QTc max was significantly correlated with LVMI (Spearman $r=0.3$, two tailed $\mathrm{p}=0.002$ ). Importantly, the relation between QTC max and LVMI was still significant (univariate analysis of variance $\mathrm{p}=0.023$ ) after adjustment for both systolic and diastolic blood pressures.

\section{QTc max and left ventricular function}

A trend was noted whereby lower ejection fractions (derived from the apical four chamber view by the method of discs) were associated with higher QTc max. The trend was not significant (Spearman $r=-0.14$, two tailed $\mathrm{p}=0.087$ ).

\section{QTc max and myocardial ischaemia}

In our study, $28 \%$ (43 of 151) of patients had significant inducible ischaemia. QTc max was not correlated with inducible myocardial ischaemia or findings on stress or rest scans (Spearman two tailed $\mathrm{p}=0.4, \mathrm{p}=0.4$, and $\mathrm{p}=0.8$, respectively). QTc max was not significantly related to smoking (univariate analysis of variance $\mathrm{p}=0.75$ ), diabetes $(\mathrm{p}=0.59)$, or total cholesterol $(\mathrm{p}=0.17)$.

\section{QTc max and HRV}

\section{Time domain data}

One hundred and eighty two patients had 24 hour ECG for analysis of HRV (time domain analysis). In our study we analysed a mean of $93 \%$ (median 97\%) of qualified sinus beats; 176 patients had $>70 \%$ analysed. This compared favourably with the minimum number required for data analysis in published studies (70-99\%). ${ }^{5}$

Abnormally low HRV as indexed by a standard deviation of normal to normal RR intervals (SDNN) $<100 \mathrm{~ms}$ was found in $20 \%$ of our patients. Stroke survivors with QTc max $>460 \mathrm{~ms}$ had a significantly higher chance of having depressed HRV as indexed by SDNN < $100 \mathrm{~ms}$ (MantelHaenszel common odds ratio 2.5, two tailed $\mathrm{p}=0.025)$. Only patients with adequate time domain data $(>70 \%$ analysed) were included in the odds ratio calculation (fig 2).

QTc max had significant negative correlations with all the time domain variables that reflected overall HRV (SDNN and 24 hour HRV triangular index), as well as long term components of HRV (standard deviation of the means of all filtered RR intervals for all five minute epochs of the analysed time period) (table 1). The residuals for QTc max were all

Table 1 Relation between maximum heart rate corrected QT interval (QTc max) and heart rate variability (HRV)

\begin{tabular}{lll}
\hline & Spearman $r$ & \multicolumn{1}{l}{$\begin{array}{l}\text { Spearman two tailed } \\
\text { p value }\end{array}$} \\
\hline 24 hour HRV triangular $-0.3(-0.3)$ & $<0.001(<0.001)$ \\
index & $-0.3(-0.3)$ & $<0.001(<0.001)$ \\
SDNN & $-0.3(-0.3)$ & $<0.001(<0.001)$ \\
SDANN & $-0.2(-0.1)$ & $0.035(0.063)$ \\
RMSSD &
\end{tabular}

Figures in brackets refer only to patients with $\geqslant 70 \%$ of data analysed ( $n=167)$.

RMSSD, square root of the mean of the sum of squares of differences between adjacent filtered RR intervals of the analysed time period: SDANN, standard deviation of the means of all filtered RR intervals for all five minute epochs of the analysed time period; SDNN, standard deviation of normal to normal RR intervals.
Table 2 Spearman correlation between QTc max and cardiac abnormalities

\begin{tabular}{lcc}
\hline & $\begin{array}{c}\text { Correlation } \\
\text { coefficient }\end{array}$ & $\begin{array}{l}\text { p Value (two } \\
\text { tailed) }\end{array}$ \\
\hline LVMI & 0.26 & 0.002 \\
Systolic BP & 0.27 & $<0.001$ \\
Diastolic BP & 0.19 & 0.007 \\
SDNN & -0.30 & $<0.001$ \\
Inducible ischaemia score & 0.063 & 0.45 \\
EF & -0.14 & 0.087 \\
\hline
\end{tabular}

BP, blood pressure; EF, ejection fraction (derived from apical four chamber view); LVMI, left ventricular mass index.

Table 3 Spearman correlation between QRS duration and cardiac abnormalities

\begin{tabular}{lcl}
\hline & $\begin{array}{l}\text { Correlation } \\
\text { coefficient }\end{array}$ & $\begin{array}{l}p \text { Value (two } \\
\text { tailed) }\end{array}$ \\
\hline LVMI & 0.17 & 0.051 \\
Systolic BP & 0.11 & 0.14 \\
Diastolic BP & 0.14 & 0.052 \\
SDNN & -0.051 & 0.50 \\
Inducible ischaemia score & 0.14 & 0.087 \\
EF & -0.11 & 0.16 \\
\hline
\end{tabular}

normally distributed (one sample Kolmogorov-Smirnov test two tailed $\mathrm{p}>0.05)$.

\section{QRS duration and cardiac abnormalities}

By way of contrast, QRS duration was not correlated with LVMI, systolic blood pressure, or SDNN (tables 2 and 3).

\section{Spectrum of cardiac abnormalities associated with prolonged QTc max}

Prolonged QTc max was thus associated with echocardiographic LVH and depressed HRV as indexed by SDNN $<100 \mathrm{~ms}$. Naturally one would expect that if a patient had both LVH and depressed HRV then QTc max would be even more prolonged. To test this hypothesis, we plotted the number of cardiac abnormalities (out of LVH and depressed HRV) against QTc max (fig 3) Thus, a patient with no LVH and good HRV had a mean QTc max of $432 \mathrm{~ms}$ (95\% confidence interval (CI) 425 to $438 \mathrm{~ms}$ ) compared with a mean of $449 \mathrm{~ms}$ (95\% CI 440 to $457 \mathrm{~ms}$ ) in patients with one abnormality (either depressed HRV or LVH) and $463 \mathrm{~ms}$

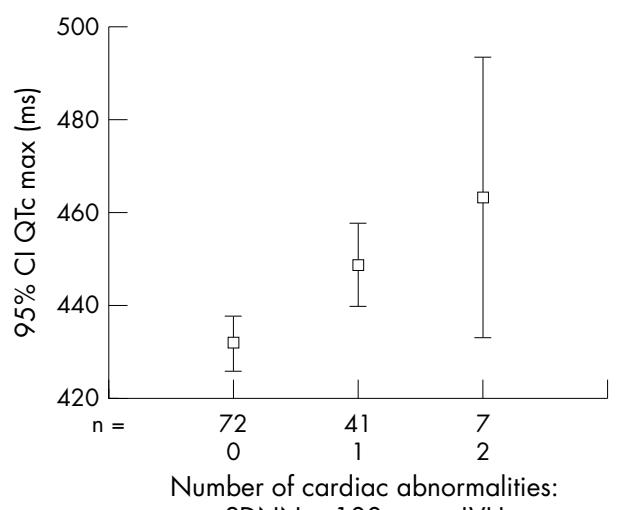

SDNN $<100$ ms or LVH

Figure 3 Error plot showing the relation between QTc max and the number of cardiac abnormalities. 
(95\% CI 443 to $483 \mathrm{~ms}$ ) in patients with both abnormalities (univariate analysis of variance $\mathrm{p}<0.001$ ).

\section{DISCUSSION}

\section{Main findings of the study}

We have shown that prolongation of QTc max was associated with increased blood pressure, LVMI, and reduced HRV. Interestingly, as the number of these cardiac abnormalities increased QTc max became more prolonged. Thus, QTc max appeared to reflect the cardiac disease burden.

In a previous observational study, ${ }^{1}$ a weak albeit significant relation was found between QTC max and some conventional risk factors of atherosclerosis such as pulse pressure, glucose, and cholesterol. However, in that study QTc max was still significantly associated with increased risk of cardiac death and total death even after adjustment for the above risk factors and for a history of symptomatic ischaemic heart disease, coronary bypass grafts, or nitrate use. The increased risk of cardiac death may be explained at least in part by the findings of the present prospective study.

\section{Clinical implications}

QTc max prolongation was associated with increased risk of cardiac death. ${ }^{1}$ This may be due to the prolongation of QTC max as the number of cardiac abnormalities increased. However, it should be noted that this was not an enormous effect. In addition to well known genes that can influence QT in long QT syndromes, milder versions of these polymorphisms may interact with acquired heart disease to produce very long QT. This may be why the correlations of QT with cardiac diseases or cardiac disease burden in the study are relatively weak. Nevertheless, it can be argued that those with prolonged QTC max now deserve more vigorous cardiac investigations to look for LVH and possibly for reduced HRV. This is because treatment and regression of $\mathrm{LVH}$ are so effective at reducing risk.

In current clinical practice, LVH is used as an indication for treatment in patients with mild hypertension even in the absence of other coronary risk factors. Importantly, LVH not only predicts death but is also reversible. ${ }^{6}$ In essential hypertension, a reduction in left ventricular mass during treatment is a favourable prognostic marker that predicts a lower risk for subsequent cardiovascular morbid events. ${ }^{7}$ This is consistent with evidence from the Framingham study, which suggests that when LVH regresses the prognosis improves. ${ }^{8}$ Indeed, if LVH regresses fully the risk appears to return to that of someone without LVH at all. To fully regress $\mathrm{LVH}$, a lower target blood pressure ought to be achieved. In light of the recent LIFE (losartan intervention for end point reduction in hypertension) study, it may be argued that stroke survivors with LVH should be offered losartan. ${ }^{9}$ However, the risk reduction in LIFE and HOPE (heart outcomes prevention evaluation) was $14-25 \%$, whereas LVH increases total risk by a factor of 2-4 times, which means that there is more room for improvement in LVH than simply using some form of angiotensin withdrawal treatment. ${ }^{10} 11$ Thus, detecting unsuspected LVH in stroke survivors may be worthwhile, since they may benefit from lower target blood pressures (as well as angiotensin withdrawal treatment, which is now standard in stroke survivors due to the PROGRESS (perindopril protection against recurrent stroke study) trial $^{12}$ ). Indeed a recent economic analysis of ours shows that such a strategy should be very cost effective, although more data are required to prove this. ${ }^{13}$

Data are accumulating to suggest that depressed HRV is a poor prognostic indicator. SDNN $<100 \mathrm{~ms}$ indicates moderately depressed HRV. In the UK-HEART (UK-heart failure evaluation and assessment of risk trial), SDNN > $100 \mathrm{~ms}$ indicated a good prognosis for patients with congestive heart failure. ${ }^{14}$ In the present study, stroke survivors with prolonged QTC max had a significantly higher chance of having depressed HRV as indexed by SDNN $<100 \mathrm{~ms}$. Further, recent evidence suggests that $\beta$ blockers and spironolactone can improve HRV. ${ }^{15-17}$ However, it should be noted that the cohorts of patients studied in the three papers quoted above had heart failure. In the present study, the lower the SDNN, the higher was the stroke survivor's heart rate (Spearman $r=-0.49$, two tailed $\mathrm{p}<0.001$ ). Further work is required to test the hypothesis that $\beta$ blockers and spironolactone can improve HRV in stroke survivors who do not have heart failure.

A further clinical implication of detecting low HRV or LVH is that non-pharmacological approaches such as physical activity should be particularly encouraged for these stroke survivors. Physical activity not only can potentially increase HRV but also has been shown to attenuate the effect of increased left ventricular mass on the risk of ischaemic stroke. $^{18}$

\section{Conclusions}

Long QTc max correlated independently with increased blood pressure, LVMI, and reduced HRV. Further, the greater the number of these cardiac abnormalities, the more prolonged was the QTc max. Thus, acquired cardiac disease may contribute to the link between a long QT and cardiac death in stroke survivors.

Patients with long QT should, therefore, in addition to having their blood pressure and electrolytes carefully monitored and corrected, be considered for echocardiography to detect treatable LVH. Future work should now concentrate on whether such a strategy of actively investigating stroke survivors with long QT would lead not only to earlier detection and treatment of $\mathrm{LVH}$ but also to reduction of their QT interval and, more important, to reduction in their risk of cardiac death. Studies in other similar populations (hypertensive LVH) suggest that such a strategy may well reduce future cardiac events, which are unfortunately particularly high among stroke survivors.

\section{ACKNOWLEDGEMENTS}

The study was supported by the British Heart Foundation.

\section{Authors' affiliations}

K Y K Wong, S McSwiggan, A Gavin, R S MacWalter, A D Struthers, University of Dundee Division of Medicine and Therapeutics, Dundee, UK

N S J Kennedy, Department of Nuclear Medicine, Ninewells Hospital and Medical School, Dundee, UK

S Y S Wong, Hypertension Research Centre, Ninewells Hospital and Medical School

Conflict of interest: none

\section{REFERENCES}

1 Wong KYK, MacWalter RS, Douglas D, et al. Long QTc predicts future cardiac death in stroke survivors. Heart 2003;89:377-81.

2 Barr CS, Naas A, Freeman M, et al. QT dispersion and sudden unexpected death in chronic heart failure. Lancet 1994;343:327-9.

3 Sahn DJ, DeMaria A, Kisslo J, et al. Recommendations regarding quantitation in M-mode echocardiography: results of a survey of echocardiographic measurements. Circulation 1978;58:1072-83.

4 European Society of Cardiology. North American Society of Pacing and Electrophysiology. Task force of the European Society of Cardiology and the North American Society of Pacing and Electrophysiology. Heart rate variability: standards of measurement, physiological interpretation, and clinical use, Circulation 1996;93:1043-65.

5 Huikuri HV, Mäkikallio T, Airaksinen KE, et al. Measurement of heart rate variability: a clinical tool or a research toy? J Am Coll Cardiol 1999:34:1878-83.

6 Perlini S, Muiesan ML, Cuspidi C, et al. Midwall mechanics are improved after regression of hypertensive left ventricular hypertrophy and normalization of chamber geometry. Circulation 2001;103:678-83. 
7 Verdecchia P, Schillaci G, Borgioni C, et al. Prognostic significance of serial changes in left ventricular mass in essential hypertension. Circulation 1998;97:48-54.

8 Kannel WB. Left ventricular hypertrophy as a risk factor in arterial hypertension. Eur Heart J 1992;13(suppl D):82-8.

9 Dahlöf B, Devereux RB, Kjeldsen SE, for the LIFE study group, et al. Cardiovascular morbidity and mortality in the losartan intervention for endpoint reduction in hypertension study (LIFE): a randomised trial against atenolol. Lancet 2002;359:995-1003.

10 Mathew J, Sleight $P$, Lonn $E$, et al. Heart Outcomes Prevention Evaluation (HOPE) Investigators. Reduction of cardiovascular risk by regression of electrocardiographic markers of left ventricular hypertrophy by the angiotensinconverting enzyme inhibitor ramipril. Circulation 2001;104:1615-21.

11 Struthers AD, Davies J. Should we add screening for and treating left ventricular hypertrophy to the management of all patients needing secondary prevention of cardiovascular disease? Q J Med 2003;96:449-52.

12 PROGRESS Collaborative Group. Randomised trial of a perindopril-based blood-pressure-lowering regimen among 6,105 individuals with previous stroke or transient ischaemic attack. Lancet. 2001;358: 1033-41, [Erratum in:Lancet 2001;358:1556, Lancet 2002;359:2120.].
13 Witham MD, Davies JI, Dawson A, et al. Hypothetical economic analysis of screening for left ventricular hypertrophy in high-risk normotensive populations. Q J M 2004;97:87-93.

14 Nolan J, Batin PD, Andrews R, et al. Prospective study of heart rate variability and mortality in chronic heart failure: results of the United Kingdom heart failure evaluation and assessment of risk trial (UK-HEART). Circulation 1998;98:1510-6.

15 Korkmaz ME, Müderrisoğlu H, Uluçam M, et al. Effects of spironolactone on heart rate variability and left ventricular systolic function in severe ischaemic heart failure. Am J Cardiol 2000;86:649-53.

16 Mortara A, La Rovere MT, Pinna GD, et al. Nonselective beta-adrenergic blocking agent, carvedilol, improves arterial baroreflex gain and heart rate variability in patients with stable chronic heart failure. J Am Coll Cardiol 2000;36:1612-8.

17 Lin JL, Chan HL, Du CC, et al. Long-term $\beta$-blocker therapy improves autonomic nervous regulation in advanced congestive heart failure: a longitudinal heart rate variability study. Am Heart J 1999;137:658-65.

18 Rodriguez CJ, Sacco RL, Sciacca RR, et al. Physical activity attenuates the effect of increased left ventricular mass on the risk of ischemic stroke: the Northern Manhattan stroke study. J Am Coll Cardiol 2002;39:1482-8.

\section{IMAGES IN CARDIOLOGY}

doi: $10.1136 /$ hrt. 2004.058560

\section{A coronary organic stenosis distal to severe, ergonovine induced spasm: decision making}

A 61 year old man was admitted to our department suffering from prolonged chest pain at rest complicated by syncope. He reported frequent episodes of typical chest pain mainly in the early morning with preserved effort tolerance. ECG recorded during chest pain revealed transient ST elevation in leads V2-V5. Coronary angiography showed irregularities of the proximal left anterior descending coronary artery (LAD) and an $80 \%$ stenosis in its middle segment (panel A). As the clinical history was suggestive of coronary spasm, we performed an intracoronary ergonovine test. After $8 \mu \mathrm{g}$ of ergonovine, the patient developed severe LAD spasm in the proximal segment associated with angina and ST segment elevation (panel B). Notably, the severity of the stenosis in the mid LAD remained unchanged. Intracoronary nitrate administration quickly resolved the spasm, symptoms, and ST changes (panel C). We decided not to perform angioplasty on the mid LAD stenosis, because of its distal location, the increased risk conferred by spasm, and the good effort tolerance. We started medical treatment with a calcium antagonist. A maximal treadmill test on treatment was negative for inducible ischaemia. At six months follow up, the patient was symptom-free and did not show transient ECG changes on Holter monitoring.

This case underlines the importance of the clinical history for the diagnosis of vasospastic angina. Furthermore, spasm occurred proximal to the site of a critical stenosis, thus suggesting that smooth muscle hyperreactivity, rather than endothelial dysfunction, is likely to play a key role in its pathogenesis.
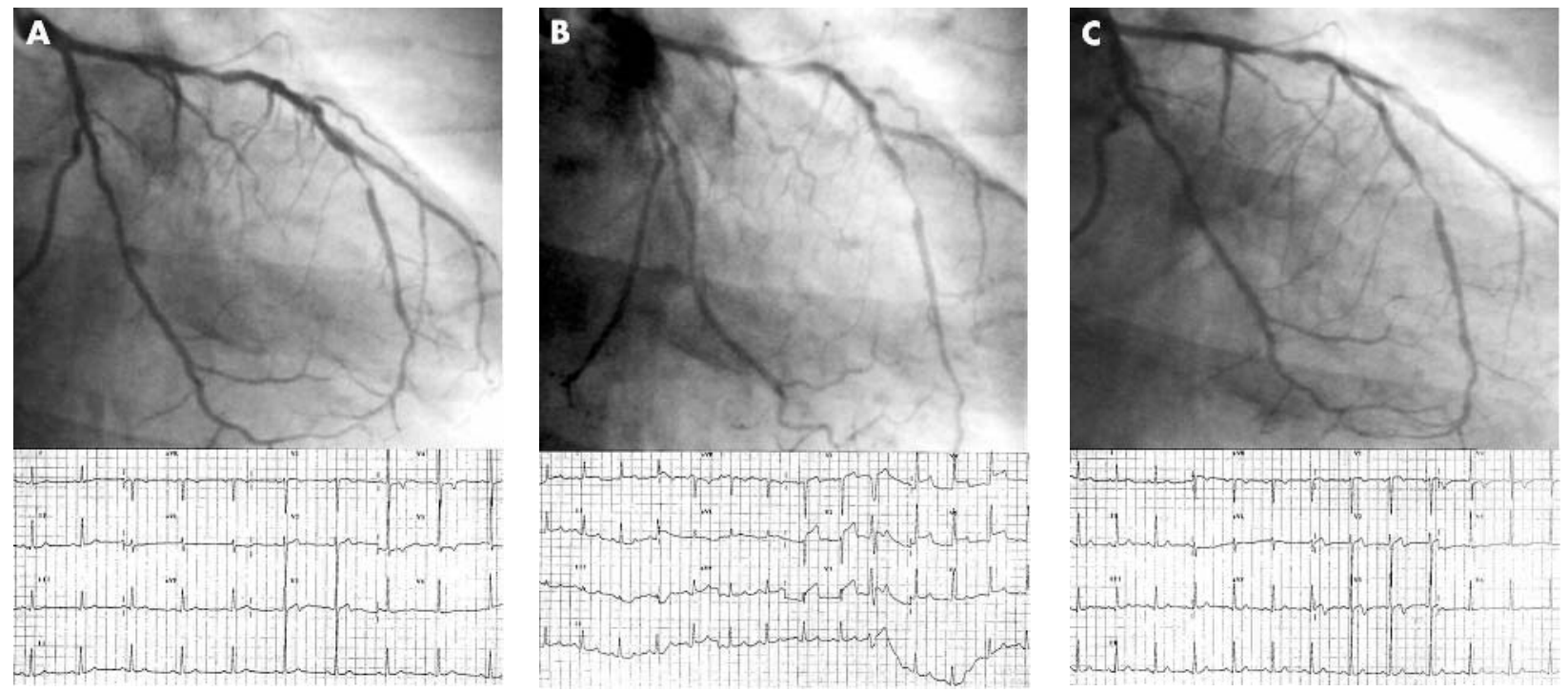